\title{
Effect of silicone oil on peripapillary capillary density in patients with rhegmatogenous retinal detachment
}

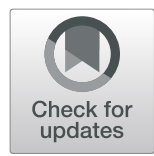

Erqian Wang ${ }^{1,2}$, Youxin Chen ${ }^{1,2}$, Ningning $\mathrm{Li}^{1,3}$ and Hanyi Min ${ }^{1,2^{*}}$ (D)

\begin{abstract}
Purpose: To evaluate the effect of silicone oil (SO) on peripapillary blood flow using OCT angiography.

Methods: This prospective case series recruited patients with unilateral rhegmatogenous retinal detachment (RRD) who underwent vitrectomy and SO tamponade. Patients were examined before SO removal and at 10 days, 1 month, and 3 months after SO removal on a spectral domain OCT angiography device (RTVue XR Avanti, Optovue Inc., CA, USA) for the measurement of radial peripapillary capillaries (RPC) vessel density (VD) in global field, superior hemifield, and inferior hemifield. Changes in RPC VD following SO removal were compared between affected eyes and contralateral eyes.

Results: Twenty-two patients were analyzed. The average duration of SO tamponade was 101.3 days (range, 90 to 119 days). After SO removal, global RPC VD increased by $1.3 \%(95 \% \mathrm{Cl}, 0.3$ to $2.3 \%)$, compared with a $-0.4 \%(95 \% \mathrm{Cl},-1.4$ to $0.7 \%)$ change in contralateral eyes $(P=0.007)$. The increase in RPC VD after SO removal mainly occurred in the superior hemifield, which was $1.6 \%(95 \% \mathrm{Cl}, 0.6$ to $2.7 \%)$. The increase in RPC VD in the inferior hemifield was $1.0 \%(95 \% \mathrm{Cl},-0.2$ to $2.1 \%$ ) after $\mathrm{SO}$ removal.

Conclusions: We detected a mild increase in peripapillary capillary density after SO removal, mainly in the superior hemifield. Our results suggested that SO tamponade could have negative effect on peripapillary blood flow, possibly by capillary compression.
\end{abstract}

Keywords: Radial peripapillary capillaries, Silicone oil, Optic neuropathy, Rhegmatogenous retinal detachment, Optical coherence tomography angiography

\section{Background}

Silicone oil (SO) tamponade, widely used in vitreoretinal surgery, is known to be associated with various complications including cataract, keratopathy, glaucoma, and optic neuropathy [1-3]. Optic neuropathy following SO tamponade, subclinical or visually significant, is reported to occur at an incidence rate varying from 0 to $28 \%[2,4]$. The

\footnotetext{
* Correspondence: minhy@pumch.cn

'Department of Ophthalmology, Peking Union Medical College Hospital, No.1 Shuai Fu Yuan, Dongcheng District, Beijing 100730, China

${ }^{2}$ Key Laboratory of Ocular Fundus Diseases, Chinese Academy of Medical Sciences, Beijing 100730, China

Full list of author information is available at the end of the article
}

pathogenesis of SO related optic neuropathy remains unclear. Possible mechanisms may include high intraocular pressure (IOP) [2] and migration of microdroplets of $\mathrm{SO}$ into optic nerve $[3,5]$. In patients who underwent vitrectomy and SO tamponade for rhegmatogenous retinal detachment (RRD) repair, optic coherence tomography (OCT) revealed retinal thickness changes, particularly in the retinal nerve fiber layers (RNFL) [6-10]. Despite the extensive studies on structural changes in optic nerve, the effect of SO on blood flow around optic nerve has not yet been studied.

Radial peripapillary capillaries (RPC), the innermost layer of capillaries that runs parallel to peripapillary

(c) The Author(s). 2020 Open Access This article is licensed under a Creative Commons Attribution 4.0 International License, which permits use, sharing, adaptation, distribution and reproduction in any medium or format, as long as you give appropriate credit to the original author(s) and the source, provide a link to the Creative Commons licence, and indicate if changes were made. The images or other third party material in this article are included in the article's Creative Commons licence, unless indicated otherwise in a credit line to the material. If material is not included in the article's Creative Commons licence and your intended use is not permitted by statutory regulation or exceeds the permitted use, you will need to obtain permission directly from the copyright holder. To view a copy of this licence, visit http://creativecommons.org/licenses/by/4.0/. The Creative Commons Public Domain Dedication waiver (http://creativecommons.org/publicdomain/zero/1.0/) applies to the data made available in this article, unless otherwise stated in a credit line to the data. 
RNFL, function to nourish RNFL in its distribution around the optic nerve head. With the advent of new imaging modality like OCT angiography, in vivo imaging of RPC becomes feasible [11], and quantitative evaluation of RPC is also available. Reduced or abnormal blood flow in RPC has been described in glaucoma and other optic nerve diseases [12-14].

Among the various conditions which need vitreoretinal surgery and SO tamponade, RRD serves as an ideal model to study the effect of SO on RPC, because the optic nerve head at presentation can be absence of intrinsic vascular abnormalities. Also, during the vitreoretinal surgery and SO removal surgery, internal limiting membrane peeling is not always necessary, therefore the likelihood of developing disassociated optic nerve fiber layer appearance [15, 16] or other retinal structural changes [17] is minimized. We thereby enrolled a prospective cohort of patients who underwent successful vitrectomy for RRD repair and subsequent SO removal, and measured RPC vessel density (VD) with $\mathrm{SO}$ and after $\mathrm{SO}$ removal. We hypothesized that SO filled eyes have transient decreased blood flow in $\mathrm{RPC}$ which will recover after SO removal. The purpose of the study was to evaluate the effect of SO on peripapillary vessel density.

\section{Methods}

This prospective, consecutive cohort study was conducted at Ophthalmology Department, Peking Union Medical College Hospital (PUMCH). This study adhered to the tenets of the Declaration of Helsinki. Ethics committee of PUMCH approved the study protocol. All participants provided written informed consent.

\section{Subjects and clinical protocols}

Patients with unilateral rhegmatogenous retinal detachment who underwent uncomplicated pars plana vitrectomy (PPV) and SO tamponade were enrolled from June 2018 to February 2019. Patients were not considered for enrollment if they had any of the following condition in either eye at presentation: 1) high myopia of over -6.00 diopters or axial length (AL) more than $26.50 \mathrm{~mm}$; 2) history of glaucoma, ischemic optic neuropathy, uveitis, or other retinal or optic nerve diseases; or 3) history of ocular surgery except for refractive and cataract surgery or 4) systemic diseases except for controlled hypertension. Patients had SO removal at least 3 months after primary vitrectomy and had follow-up visits at 10 days, 1 month, and 3 months after SO removal. Patients were excluded from analysis if they: 1) failed to complete follow-up; 2) had recurrent retinal detachment which needed secondary surgical repair; 3) had SO removal combined with epiretinal membrane peeling or internal limiting membrane peeling which might cause iatrogenic RNFL and RPC changes; or 4) had insufficient OCT angiography signal strength (scan quality index $<6 / 10$ ) due to severe cataract at any follow-up visit.

We also collected the following clinical characteristics: 1) macula status at presentation; 2) whether cataract surgery was performed in combined with $\mathrm{SO}$ removal; 3) duration of SO tamponade; and 4) whether SO tamponade was full indicated by whether SO-water interface was invisible under indirect ophthalmoscope after pupil dilation.

\section{Surgical techniques}

All patients had standard 25G PPV and SO tamponade under retrobulbar anesthesia. One single surgeon (H.M.) performed all surgical procedures using Constellation Vision System (Alcon Laboratories, Inc., Texas, USA) and $25 \mathrm{G}$ trocar cannula system. The IOP was set at $25 \mathrm{mmHg}$ during surgery. Retinal detachment was repaired in a standard fashion. Briefly, following vitreous removal, vitreoretinal traction release, subretinal fluid drainage and endolaser retinopexy of the retinal breaks, silicone oil (Oxane 5700, Bausch \& Lomb, Rochester, N.Y., USA) was instilled. In all patients, the retina was completely reattached at the end of surgery. At the primary vitrectomy surgery, we did not perform combined cataract surgery because none of the study subjects had visually significant cataract. The major concern of performing cataract surgery at primary surgery was that the gradual opacification of lens capsule at the capsulorhexis margin might interfere with adequate peripheral retina examination before $\mathrm{SO}$ removal. Another concern was that removing cataract might increase the risk of SO migration into the anterior chamber in eyes with zonular weakness. Prone position was adopted within 2 weeks after vitrectomy. Following vitrectomy surgeries, patients used topical antibiotics, corticosteroid, and IOP-lowering medications in case of need.

The same surgeon (H.M.) performed SO removal surgeries using Constellation Vision System and 23G trocar cannula system, with IOP set at $25 \mathrm{mmHg}$. Fluid-air exchange was done to eliminate residual SO droplets. Cataract surgery was performed in phakic eyes of patients over 60 years old and in eyes with visually significant cataract. In these patients, phacoemulsification cataract removal was performed before silicone oil removal, and intraocular lens was implanted after silicone oil removal. Scleral wound was sutured in all patients. Topical antibiotics and corticosteroid were applied and tapered off within 4 weeks after SO removal.

\section{OCT angiography imaging and processing}

For optic disk OCT angiography scan we used a commercially available spectral domain OCT angiography device RTVue XR Avanti (Optovue Inc. Fremont, CA, USA). This device uses light source of $840 \mathrm{~nm}$ and operates at scan speed of 70,000 A-scans per second. Both 
the affected eye and the healthy contralateral eye were imaged on a $4.5 \mathrm{~mm} \times 4.5 \mathrm{~mm}$ optic disc scan via dilated pupil. Two consecutive scans were performed and the one with optimal image quality and without motion artifacts were used for analysis. All image acquisitions were made by one single investigator (E.W.).

For quantitative assessment of RPC VD, we used a built-in software AngioVue AngioAnalytics (Version 2017.1, Optovue Inc. Fremont, CA, USA). This software uses a split-spectrum amplitude decorrelation angiography algorithm with three-dimensional projection artifact removal technique and provides selective information on capillary VD in the RPC layer. By the definition of the software, peripapillary area is the area between the $2 \mathrm{~mm}$ and $4 \mathrm{~mm}$ diameter annular contour lines around disc margin. Manual adjustments of disc contour and layer segmentation were made in case of need. This software automatically generated the RPC VD of the global 360-degree area, the superior hemifield, and the inferior hemifield, based on Garway-Heath's map (Fig. 1) [18]. The OCT angiography scan quality index (from 1 to 10) was also provided by the software.

\section{Outcome measures}

Primary outcome was the change in global RPC VD from before SO removal to 3 months after SO removal, compared with the contralateral eyes.

Secondary outcomes included: 1) the change in RPC VD at 3 months after SO removal in superior and inferior hemifield, compared with the contralateral eyes; 2) the trend of changes in global RPC VD before SO removal and at 10 days, 1 month, and 3 months after SO removal; and 3) the association between global RPC VD change and age, sex, AL, preoperative macula status, combined cataract surgery with $\mathrm{SO}$ removal, SO tamponade duration, as well as full SO tamponade.

We conducted sensitivity analysis by comparing the change in global RPC VD between affected eyes and contralateral eyes in a subgroup of patients who did not have cataract surgery during $\mathrm{SO}$ removal, to eliminate the confounding effect from signal strength improvements due to cataract removal.

\section{Statistical analysis}

We analyzed data using SPSS program version 22.0 (IBM, Inc., Chicago, IL). Kolmogrov-Smirnov method was used for test of normality. In the comparison of demographic and clinical characteristics between affected and contralateral eyes, we used Wilcoxon signed ranks test and Fisher exact test for continuous and dichotomous variables, respectively. In the comparison of RPC VD changes between affected and contralateral eyes, we used mixed effect model. We evaluated the main effect of SO removal surgery on RPC VD change, with the changes in IOP and OCT angiography scan quality index adjusted as covariates. The trend of changes in RPC VD after SO removal was analyzed using repeated measures ANOVA (analysis of variance) and Bonferroni's post hoc test. Pearson or Spearman correlation analysis, where appropriate, was used to study RPC VD change for its association with

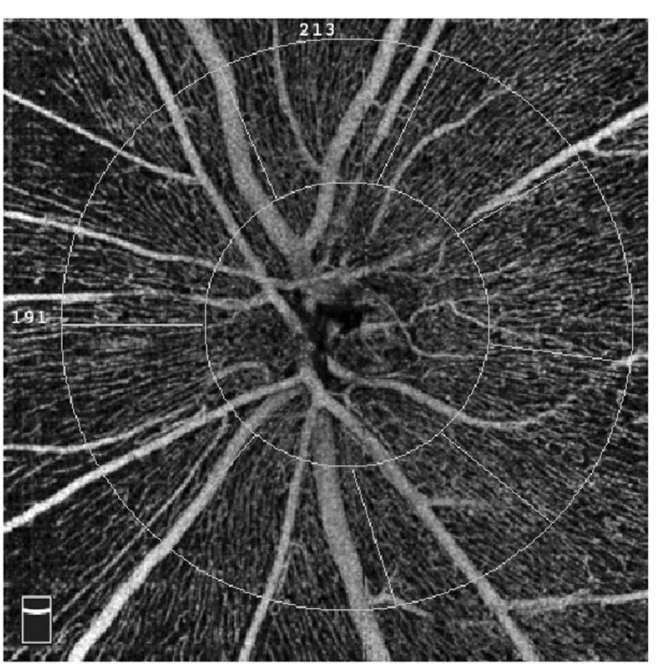

\begin{tabular}{|c|c|c|}
\hline \multicolumn{3}{|c|}{ Capillary Density \& RNFL Thickness } \\
\hline Density (\%) & Section & Thickness $(\mu \mathrm{m})$ \\
\hline 49.1 & Whole Image & N/A \\
\hline 50.9 & Inside Disc & N/A \\
\hline$\rightarrow 52.1$ & Peripapillary & 102 \\
\hline$* 52.2$ & - Superior-Hemi & 92 \\
\hline$\Delta 52.1$ & - Inferior-Hemi & 112 \\
\hline 48.0 & - Nasal Superior & 93 \\
\hline 46.2 & - Nasal Inferior & 83 \\
\hline 50.0 & - Inferior Nasal & 135 \\
\hline 57.1 & - Inferior Tempo & 155 \\
\hline 56.7 & - Tempo Inferior & 77 \\
\hline 55.9 & - Tempo Superior & 66 \\
\hline 54.2 & - Superior Tempo & 91 \\
\hline 51.6 & - Superior Nasal & 120 \\
\hline
\end{tabular}

Fig. 1 Measuring radial peripapillary capillaries (RPC) vessel density (VD) based on Garway-Heath's map. (Left) The representative $4.5 \mathrm{~mm} \times 4.5 \mathrm{~mm}$ en face OCT angiography image is centered on optic nerve head. The peripapillary area is overlaid by annular contour lines with $2 \mathrm{~mm}$ and $4 \mathrm{~mm}$ diameter around disc margin. The peripapillary area is further divided into eight sectors according to Garway-Heath's map. (Right) The built-in software automatically calculates RPC VD based on Garway-Heath's map. The global RPC VD (arrow) is measured from the 360-degree annular peripapillary area. The RPC VD of the superior (asterisk) and inferior (triangle) hemifield is measured from the superior four sectors and the inferior four sectors, respectively 
demographic and clinical characteristics. A $P$ value of less than 0.05 was considered statistically significant.

\section{Results}

\section{Demographic and clinical characteristics}

The study enrolled 31 patients. After excluding 9 patients (3 lost to follow-up after SO removal, 3 had poor OCT angiography image quality before $\mathrm{SO}$ removal due to severe cataract, 2 had internal limiting membrane peeling for macular hole repairment or subfoveal residual perfluorocarbon removal, and 1 had scleral buckling for recurrent retinal detachment), we included 22 patients for analysis. The average duration of SO tamponade was 101.3 days (range, 90 to 119 days). The demographic and clinical characteristics are summarized in Table 1.

\section{Peripapillary vessel density}

In the affected eyes, the scan quality index before and 90 days after SO removal was $7.2 \pm 1.0$ and $7.8 \pm 1.0$, respectively. The mean change in scan quality index was $0.6(95 \% \mathrm{CI},-0.1$ to $1.3, P=0.11)$. The scan quality index in the contralateral control eyes did not change after SO removal $(8.1 \pm 1.0$ versus $8.1 \pm 1.2, P=0.80)$. The global RPC VD increased after SO removal (Table 2). The average change in global RPC VD was $1.3 \%(0.3$ to $2.3 \%)$ and $-0.4 \%(95 \% \mathrm{CI},-1.4$ to $0.7 \%)$ in affected and contralateral eyes, respectively. Mixed effect model analysis revealed that the difference in RPC VD change between affected and contralateral eyes was 1.7\% (95\%CI, 0.4 to $3.0 \%$ ), which was statistically significant $(P=0.007)$ after adjusting for the changes in IOP and OCT angiography scan quality index.

The increase of RPC VD after SO removal mainly occurred in the superior hemifield, which was $1.6 \%$ (95\% CI, 0.6 to $2.7 \%$ ). The increase of RPC VD in the inferior hemifield was $1.0 \%$ (95\%CI, -0.2 to $2.1 \%$ ) after SO removal (Table 2).
There was a significant trend of increase in global RPC VD after SO removal $(F=5.417, P=0.002)$ (Fig. 2).

No association was found between the change in global RPC VD and age $(P=0.61)$, sex $(P=0.71)$, refractive error $(P=0.87)$, axial length $(P=0.51)$, preoperative macular status $(P=0.38)$, combined cataract surgery during SO removal $(P=1.00)$, duration of SO tamponade $(P=0.63)$, or full SO tamponade $(P=0.76)$.

In 13 patients who did not have cataract surgery during SO removal, the scan quality index in the affected eyes before and 90 days after SO removal was $7.3 \pm 1.1$ and $7.9 \pm 0.9$, respectively. The mean change in scan quality index was $0.5(95 \% \mathrm{CI},-0.5$ to $1.5, P=0.27)$. The scan quality index in the contralateral control eyes did not change after SO removal $(7.9 \pm 1.1$ versus $8.0 \pm$ $1.2, P=0.34$ ). In the affected eyes, the global RPC VD increased by $1.4 \%(95 \% \mathrm{CI}, 0.2$ to $2.5 \%)$ after SO removal, although mixed effect model revealed no statistically significant difference $(P=0.08)$ compared with the $-0.0 \%$ $(95 \% \mathrm{CI},-1.4$ to $1.3 \%)$ change in contralateral eyes.

\section{Discussion}

To our knowledge, the current study is the first one that investigated the effect of $\mathrm{SO}$ on peripapillary blood flow. We found a mild but definite increase of RPC VD in SO filled eyes following $\mathrm{SO}$ removal. Our results suggested that SO may have potential negative effect on microvascular blood flow around optic nerve head.

The increase in RPC VD reflected a mild but true increase in microvascular blood flow around optic nerve head after SO removal. The repeatability of OCT angiography measurements had been well demonstrated in previous studies $[19,20]$. Factors that could influence the accuracy of OCT angiography measurements included IOP fluctuation [21], refractive media opacity and insufficient signal strength [20,22, 23], diurnal variations and blood pressure changes [24]. In our study, we made several efforts to limit confounding factors. First, with

Table 1 Demographic and Clinical Characteristics $(n=22)$

\begin{tabular}{llll}
\hline Characteristics & Affected eye & Contralateral eye $^{*}$ & \\
\hline Age (Years) & $52.2 \pm 12.3$ & & - \\
Sex (Male/Female) & $15 / 7$ & - \\
Refractive Error (Diopters) & $-2.5 \pm 2.3$ & $-2.4 \pm 2.3$ & $24.79 \pm 1.29$ \\
Axial Length (mm) & $24.81 \pm 1.24$ & $\mathrm{NA}$ & $\mathrm{N}$ \\
Macula Status (On/Off) & $3 / 19$ & $\mathrm{NA}$ & $\mathrm{NA}$ \\
SO Removal Combined with Cataract Surgery (Yes/No) & $9 / 13$ & $\mathrm{NA}$ & $\mathrm{NA}$ \\
SO Tamponade Duration (Days) & $101.3 \pm 8.4$ & $\mathrm{NA}$ & $\mathrm{NA}$ \\
Full SO Tamponade $^{\text {(Yes/No) }}$ & $15 / 7$ & & $\mathrm{NA}$ \\
\hline
\end{tabular}

Age, refractive error, and axial length are shown as mean \pm standard deviation

${ }^{*} P$ values are from Wilcoxon signed ranks test for continuous variables

${ }^{a}$ Silicone oil tamponade is deemed as full if oil-water interface is not visible under indirect ophthalmoscope after pupil dilation

SO silicone oil, NA not applicable 
Table 2 Comparison of Peripapillary Vessel Density Changes After Silicone Oil Removal Between Affected and Contralateral Eyes $(n=22)$

\begin{tabular}{|c|c|c|c|}
\hline Measurements & & Affected eyes & Contralateral eyes \\
\hline \multirow[t]{4}{*}{ Global RPC VD } & Before SOR & $46.8 \pm 3.7 \%$ & $51.0 \pm 3.3 \%$ \\
\hline & After SOR & $48.1 \pm 3.8 \%$ & $50.6 \pm 3.4 \%$ \\
\hline & Change & $1.3 \%$ (0.3 to $2.3 \%)$ & $-0.4 \%(-1.4$ to $0.7 \%)$ \\
\hline & Difference of change ${ }^{a}$ & \multicolumn{2}{|c|}{$1.9 \%(0.6$ to $3.2 \%), P=0.007$} \\
\hline \multirow[t]{4}{*}{ Superior RPC VD } & Before SOR & $46.8 \pm 3.9 \%$ & $51.1 \pm 3.8 \%$ \\
\hline & After SOR & $48.4 \pm 3.6 \%$ & $50.8 \pm 3.8 \%$ \\
\hline & Change & $1.6 \%$ (0.6 to $2.7 \%)$ & $-0.4 \%(-1.4$ to $0.7 \%)$ \\
\hline & Difference of change ${ }^{a}$ & \multicolumn{2}{|c|}{$2.2 \%(0.9$ to $3.4 \%), P=0.002$} \\
\hline \multirow[t]{4}{*}{ Inferior RPC VD } & Before SOR & $46.7 \pm 4.3 \%$ & $50.8 \pm 3.2 \%$ \\
\hline & After SOR & $47.7 \pm 4.4 \%$ & $50.4 \pm 3.5 \%$ \\
\hline & Change & $1.0 \%(-0.2$ to $2.1 \%)$ & $-0.4 \%(-1.5$ to $0.7 \%)$ \\
\hline & Difference of change ${ }^{a}$ & \multicolumn{2}{|c|}{$1.6 \%(-0.0$ to $3.2 \%), P=0.06$} \\
\hline
\end{tabular}

Vessel densities before and after silicone oil removal are described as mean \pm SD

Changes in vessel densities are described as mean ( $95 \%$ confidence interval)

a Difference of change in vessel densities between affected and contralateral eyes was analyzed using mixed model analysis and presented as mean (95\% confidence interval). The $P$ value represents the main effect of SOR with changes in intraocular pressure and OCT angiography image quality score adjusted as covariates

RPC Radial Peripapillary Capillaries, VD Vessel Density, SOR Silicone Oil Removal

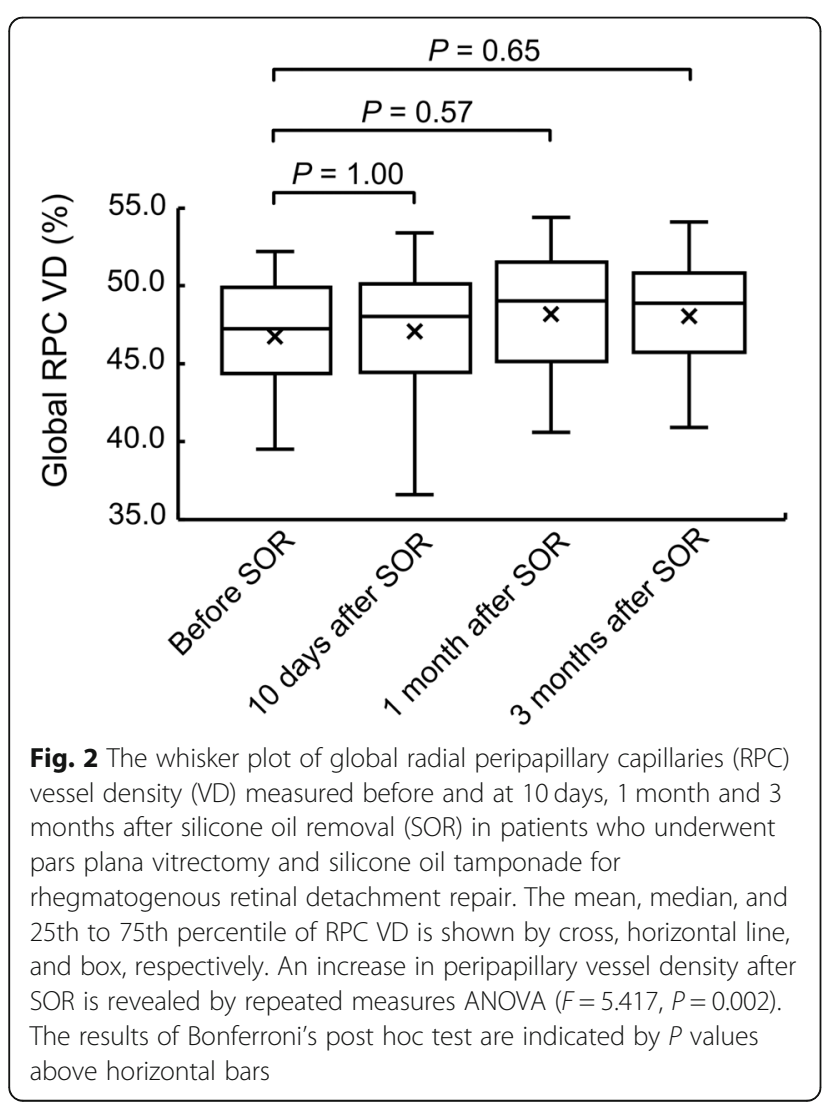

healthy fellow eyes serving as controls, the impact of systemic errors, such as diurnal variations and systemic circulatory changes, could be minimized. Second, the main outcome measures were acquired at least 3 months after primary vitrectomy and 3 months after SO removal, which was a long enough period for acute surgical inflammation and IOP fluctuations to subside. Thus, the resulting RPC VD measurements were unlikely to be affected by postoperative corneal edema, anterior chamber flares, hypotonic papilledema, or potentially other unusual conditions. Third, we excluded subjects with severe cataract before SO removal and included only patients with adequate OCT angiography image quality. Also, the sensitivity analysis in patients who did not have cataract surgery during SO removal detected similar increase in RPC VD. Therefore, the increase in peripapillary vessel density was unlikely to be confounded by improvement in optic media clarity due to cataract removal. Fourth, although OCT angiography scan quality index exhibited mild increase after SO removal, its changes were adjusted as covariates when comparing RPC VD changes between affected eyes and controls. Fifth, trend analysis revealed that RPC VD after SO removal manifested a steady increase rather than an irregular fluctuation, supporting the reliability of our results. One might argue that the recovery of peripapillary vessel density could reflect a continuous improvement of optic nerve microcirculation after retinal reattachment. However, peripapillary vessel density increase was not observed from 1 month to 3 months after primary vitrectomy surgery $(46.7 \pm 4.0 \%$ versus $46.8 \pm$ 
$3.7 \%, P=0.92$, paired $t$ test), suggesting that the increase of RPC VD after SO removal was not part of a prolonged recovery process from retinal reattachment. One might also argue that $\mathrm{SO}$ tamponade and refractive changes after SO removal will affect image magnification which will further influence RPC VD measurements. By comparing the disk diameter on en-face OCT images before and after SO removal $(1.518 \pm 0.226 \mathrm{~mm}$ and $1.518 \pm 0.229 \mathrm{~mm}, P=1.00$ ), we did not detect significant difference in image magnification with and without SO tamponade. Therefore, SO tamponade and refractive changes are unlikely to significantly influence RPC VD measurements. Others might argue that OCT angiography images under SO could be dark because of masking artifact. However, high quality images acquisition could be achieved in SO filled eyes when a suitable angle of scan could be found to avoid silicone oil reflection. Based on the above considerations, the increase in RPC VD was likely to be secondary to SO removal instead of other confounders. Although the amount of RPC VD increase was small, we are confident that a real improvement of peripapillary capillary density occurred after the removal of SO.

The superior hemifield of RPC showed a more prominent increase in VD than the inferior hemifield, suggesting that SO might affect the superior peripapillary microvasculatures more than the inferior vessels. One possible explanation was that the buoyancy of silicone oil exerted compression on peripapillary capillaries. The specific gravity of standard SO is lower than intraocular fluid. This physical property allows for a strong ability to tamponade the superior peripheral retina, while the lower periphery may not be efficiently supported. Although SO can support the area around optic disc and the larger posterior pole, we postulate that the superior hemifield of RPC might have received greater compression from SO tamponade. Interestingly, unlike RPC VD, the global RNFL thickness change at 3 months after SO removal did not show significant difference between affected and contralateral eyes ( $95 \% \mathrm{CI},-3.1 \mu \mathrm{m}$ to $6.7 \mu \mathrm{m}$, $P=0.47$, mixed effect model). This indicated that the retinal nerve fibers might not be as vulnerable to mechanical compression as peripapillary capillaries were.

We tried to examine global RPC VD change after SO removal for its association with demographic and clinical characteristics including duration of SO tamponade and full SO tamponade, but none of these factors was associated with RPC VD change. Several reasons might account for the negative findings. First, the judgement of full SO tamponade relied on not only the quantity of SO in eye, but also the skill of examiner and the diameter of dilated pupil. Second, duration of SO tamponade was 3 to 4 months according to our institute's common practice. If SO tamponade was kept for a longer duration, we might be able to detect a positive correlation between RPC VD change and SO tamponade duration. According to previous studies, longer duration of SO tamponade might be related with retinal structural abnormalities [25]. Third, the small sample size might be insufficient to yield significant results in correlation analysis.

In the current study, we did not evaluate RPC VD changes before and after primary vitrectomy and $\mathrm{SO}$ tamponade. Several difficulties existed in such investigation. First, most RRD patients at presentation had low vision, poor fixation, and considerable vitreous opacity which reduced the quality OCT angiography images. In some patients, the peripapillary retina was even detached, resulting in segmentation errors in OCT angiography images which could hardly be fully adjusted. Therefore, the accurate evaluation of RPC VD before primary vitrectomy and SO tamponade was difficult. Second, to evaluate the solitary effect of SO tamponade on peripapillary vessel density and eliminate the confounding effect from surgical trauma of primary vitrectomy, a group of controls who underwent vitrectomy without SO tamponade would be needed. However, legal SF6 or C3F8 product for medical use was unavailable across China until September 2019 when we end the current study. Considering the above difficulties, we studied the effect of $\mathrm{SO}$ on peripapillary vessel density by comparing RPC VD before and after SO removal, based on the assumption that SO removal without retinal manipulations caused limited surgical trauma and minimal pathophysiological changes in the posterior segment of eye. Previous studies had compared the effect of different types of tamponade on retinal structures using OCT. A case series by Christensen et al. [7] performed OCT scans in RRD patients and detected inner retinal thinning in SO filled eyes compared with gas filled eyes. On the contrary, a case control study by Bansal et al. [26] suggested that optic neuropathy after vitrectomy was related with intraoperative systemic hypotension and low ocular perfusion, but not related with the type of tamponade. Further studies are needed to compare peripapillary vessel density changes in eyes with $\mathrm{SO}$ and gas tamponade.

The strengths of this study included prospective design, longitudinal follow-up, and high-quality data acquisition. We acknowledge several limitations in our study. First, we excluded patients with high myopia whose peripapillary microvasculatures might react differently to the use of SO. Second, our findings should be limited to RRD. We should be careful about generalizing to other vitreoretinal diseases. Third, the duration of SO tamponade was short, and the effect of long-term SO tamponade on peripapillary vessel density remained unknown. Fourth, we did not compare the effect of SO and gas tamponade on RPC VD changes, as was discussed above. 
Future studies should further examine this issue. Fifth, we only identified subclinical increase in peripapillary vessel density after SO removal, and the increase was not analyzed for its visual correlation.

\section{Conclusions}

In patients who underwent vitrectomy and SO tamponade for RRD repair, we detected a mild increase in peripapillary capillary vessel density after SO removal, mainly in the superior hemifield of peripapillary area. Our findings suggested that $\mathrm{SO}$ removal was associated with a recovery of peripapillary microvascular blood flow, possibly due to the release of vascular compression. Our results implied that the use of SO should be held with caution due to its potential negative impact on optic nerve microcirculation, even when the duration of SO tamponade does not exceed four months. Our study also added new insights to the complex biologic mechanisms of SO related optic neuropathy.

\section{Abbreviations}

AL: Axial length; ANOVA: Analysis of variance; IOP: Intraocular pressure; NA: Not applicable; OCT: Optical coherence tomography; RPC: Radial peripapillary capillaries; SO: Silicone oil; SOR: Silicone oil removal; VD: Vessel density; RNFL: Retinal nerve fiber layers; PPV: Pars plana vitrectomy

\section{Acknowledgements}

The authors acknowledge Dr. Yanlong Li for his statistical support.

\section{Authors' contributions}

EW, YC and HM designed the study. HM and NL performed all surgeries. EW and NL collected and analyzed all data. EW, YC, NL and HM interpreted the data and results. EW drafted the manuscript. YC, NL, and HM reviewed the manuscript. All authors read and approved the final manuscript.

\section{Authors' information}

Null.

\section{Funding}

The study was supported by the Non-Profit Central Research Institute Fund of Chinese Acadamy of Medical Science (Grant No. 2018PT32029) and the Innovation Fund for Medical Sciences of Chinese Acadamy of Medical Science (Grant No. 2017-I2M-1-012). The funding organization had no role in the design or conduct of this research.

\section{Availability of data and materials}

The datasets used and/or analysed during the current study are available from the corresponding author on reasonable request.

\section{Ethics approval and consent to participate}

The study was in compliance with the tenets of the Declaration of Helsinki. The Ethics Committee of Peking Union Medical College Hospital approved the study protocol. All participants gave informed consent.

\section{Consent for publication}

Not applicable.

\section{Competing interests}

The authors declare that they have no competing interests.

\section{Author details}

'Department of Ophthalmology, Peking Union Medical College Hospital, No.1 Shuai Fu Yuan, Dongcheng District, Beijing 100730, China. ${ }^{2}$ Key Laboratory of Ocular Fundus Diseases, Chinese Academy of Medical Sciences,
Beijing 100730, China. ${ }^{3}$ Department of Operating Room, Peking Union Medical College Hospital, Beijing 100730, China.

Received: 22 April 2020 Accepted: 26 June 2020

Published online: 07 July 2020

\section{References}

1. Federman $J$, Schubert HD. Complications associated with the use of silicone oil in 150 eyes after retina-vitreous surgery. Ophthalmology. 1988; 95:870-6.

2. Morphis $\mathrm{G}$, Irigoyen $\mathrm{C}$, Eleuteri $\mathrm{A}$, et al. Retrospective review of 50 eyes with long-term silicone oil tamponade for more than 12 months. Graefes Arch Clin Exp Ophthalmol. 2012;250:645-52.

3. Grzybowski A, Pieczynski J, Ascaso FJ. Neuronal complications of intravitreal silicone oil: an updated review. Acta Ophthalmol. 2014;92:201-4.

4. Shalchi Z, Mahroo OA, Shunmugam M, et al. Spectral domain optical coherence tomography findings in long-term silicone oil-related visual loss. Retina. 2015:35:555-63.

5. Knecht P, Groscurth P, Ziegler U, et al. Is silicone oil optic neuropathy caused by high intraocular pressure alone? A semi-biological model. $\mathrm{Br} J$ Ophthalmol. 2007;91:1293-5.

6. Takkar B, Azad R, Kamble N, Azad S. Retinal nerve fiber layer changes following primary retinal detachment repair with silicone oil tamponade and subsequent oil removal. J Ophthalmic Vis Res. 2018;13:124-9.

7. Christensen UC, la Cour M. Visual loss after use of intraocular silicone oil associated with thinning of inner retinal layers. Acta Ophthalmol. 2012;90: 733-7.

8. Rabina G, Azem N, Barequet D, et al. Silicone oil tamponade effect on macular layer thickness and visual acuity. Retina. 2019. https://doi.org/10. 1097/IAE.0000000000002464

9. Jurisic D, Geber MZ, Cavar I, Utrobicic DK. Retinal layers measurements following silicone oil tamponade for retinal detachment surgery. Semin Ophthalmol. 2018:33:711-8.

10. Zoric Geber M, Bencic G, Vatavuk Z, Ivekovic R, Friberg TR. Retinal nerve fibre layer thickness measurements after successful retinal detachment repair with silicone oil endotamponade. Br J Ophthalmol. 2015;99:853-8.

11. Spaide RF, Klancnik JM, Cooney MJ. Retinal vascular layers imaged by fluorescein angiography and optical coherence tomography angiography. JAMA Ophthalmol. 2015:133:45-50.

12. Mastropasqua R, Agnifili L, Borrelli E, et al. Optical coherence tomography angiography of the peripapillary retina in normal-tension glaucoma and chronic nonarteritic anterior ischemic optic neuropathy. Curr Eye Res. 2018; 43:778-84.

13. Mansoori T, Sivaswamy J, Gamalapati JS, Balakrishna N. Radial peripapillary capillary density measurement using optical coherence tomography angiography in early glaucoma. J Glaucoma. 2017;26:438-43.

14. Augstburger E, Zeboulon P, Keilani C, Baudouin C, Labbe A. Retinal and choroidal microvasculature in nonarteritic anterior ischemic optic neuropathy: an optical coherence tomography angiography study. Invest Ophthalmol Vis Sci. 2018:59:870-7.

15. Runkle AP, Srivastava SK, Yuan A, et al. Factors associated with development of dissociated optic nerve fiber layer appearance in the pioneer intraoperative optical coherence tomography study. Retina. 2018;38(Suppl 1):S103-9.

16. Mitamura Y, Ohtsuka K. Relationship of dissociated optic nerve fiber layer appearance to internal limiting membrane peeling. Ophthalmology. 2005; 112:1766-70.

17. Hisatomi T, Tachibana T, Notomi S, et al. Internal limiting membrane peeling-dependent retinal structural changes after vitrectomy in rhegmatogenous retinal detachment. Retina. 2018;38:471-9.

18. Ferreras A, Pablo LE, Garway-Heath DF, Fogagnolo P, Garcia-Feijoo J. Mapping standard automated perimetry to the peripapillary retinal nerve fiber layer in glaucoma. Invest Ophthalmol Vis Sci. 2008;49:3018-25.

19. Lim CW, Cheng J, Tay ELT, et al. Optical coherence tomography angiography of the macula and optic nerve head: microvascular density and test-retest repeatability in normal subjects. BMC Ophthalmol. 2018;18: 315.

20. Venugopal JP, Rao HL, Weinreb RN, et al. Repeatability of vessel density measurements of optical coherence tomography angiography in normal and glaucoma eyes. Br J Ophthalmol. 2018;102:352-7. 
21. Hollo G. Influence of large intraocular pressure reduction on peripapillary $\mathrm{OCT}$ vessel density in ocular hypertensive and glaucoma eyes. J Glaucoma. 2017;26:e7-10

22. Lim HB, Kim YW, Kim JM, Jo YJ, Kim JY. The importance of signal strength in quantitative assessment of retinal vessel density using optical coherence tomography angiography. Sci Rep. 2018;8:12897.

23. Chang R, Nelson AJ, LeTran V, et al. Systemic determinants of peripapillary vessel density in healthy African Americans: the African American eye disease study. Am J Ophthalmol. 2019;207:240-7.

24. Muller VC, Storp JJ, Kerschke L, et al. Diurnal variations in flow density measured using optical coherence tomography angiography and the impact of heart rate, mean arterial pressure and intraocular pressure on flow density in primary open-angle glaucoma patients. Acta Ophthalmol. 2019; 97:e844-9.

25. Durrani AK, Rahimy E, Hsu J. Outer retinal changes on spectral-domain optical coherence tomography pre- and post-silicone oil removal. Ophthalmic Surg Lasers Imaging Retina. 2017:48:978-82.

26. Bansal AS, Hsu J, Garg SJ, et al. Optic neuropathy after vitrectomy for retinal detachment: clinical features and analysis of risk factors. Ophthalmology 2012:119:2364-70

\section{Publisher's Note}

Springer Nature remains neutral with regard to jurisdictional claims in published maps and institutional affiliations.

Ready to submit your research? Choose BMC and benefit from:

- fast, convenient online submission

- thorough peer review by experienced researchers in your field

- rapid publication on acceptance

- support for research data, including large and complex data types

- gold Open Access which fosters wider collaboration and increased citations

- maximum visibility for your research: over $100 \mathrm{M}$ website views per year

At BMC, research is always in progress.

Learn more biomedcentral.com/submissions 